\title{
THE HETEROGENEITY PRINCIPLE
}

\author{
Ronald R. Knipling ${ }^{1}$, Anders E. af Wåhlberg ${ }^{2}$ \\ ${ }^{1}$ Safety for the Long Haul Inc., Arlington, Virginia, USA \\ Email: rknipling@verizon.net \\ ${ }^{2}$ Cranfield University, Bedfordshire, United Kingdom \\ Email: A.E.Af-Wahlberg@cranfield.ac.uk
}

\begin{abstract}
Summary: The theoretical foundation of the Naturalistic Driving (ND) MixedSafety-Critical Event (SCE) methodology is found in the historical writings of H.W. Heinrich, a $20^{\text {th }}$ century industrial safety engineer. Heinrich espoused the theory that serious accidents, minor ones, and even no-injury operator errors all had identical or highly similar causal mechanisms and that accident consequences were essentially unlinked to causes. This became the basis for today's ND MixedSCE method, whereby a variety of mostly non-crash avoidance maneuvers (e.g., hard braking, swerves) and other kinematic events (e.g., lane drifts) are aggregated by researchers to form a dependent variable dataset ostensibly representative of important and harmful crashes. This paper examines this approach and finds it to be invalidated by the pervasive causal heterogeneity of crashes and would-be surrogates. Crashes are heterogeneous horizontally by type and vertically by severity. This paper argues instead for the heterogeneity principle as the foundational assumption and guiding tenet for any effort to extrapolate causal evidence from surrogates (e.g., non-crashes or minor crashes) to a different and more important target crash population such as serious crashes.
\end{abstract}

\section{INTRODUCTION: H. W. HEINRICH'S STATURE IN INDUSTRIAL SAFETY}

H. W. Heinrich was an influential mid-20 $0^{\text {th }}$ century industrial safety engineer. Based on studies of military and industrial accidents, Heinrich (1941) formulated several premises regarding accident causation and prevention. The "Heinrich Triangle" is a schematic illustrating the common relationship between accident severity and frequency. An implication of the triangle, generally corroborated, was that an effective approach to preventing serious accidents is to monitor and reduce the occurrence of minor ones and the at-risk behaviors precipitating them. This principle has been a basis for behavior-based safety and, in trucking, onboard safety monitoring of driver behaviors (Pradhan et al., 2017; af Wåhlberg, 2008).

Current thought in industrial safety, however, seems to be that Heinrich's Triangle has been oversold as a concept for reducing serious accidents. The National Safety Council (Manuele, 2014) has criticized Heinrich both in regard to accident reduction and to causal analysis. Manuele $(2014,2008)$ cites industrial examples where minor injuries were reduced far more effectively than major injuries by behavioral safety interventions. Dunlop (2013) also cites evidence against the "general belief that it is possible to avoid major injuries or incidents by preventing minor ones." Her view is that an industrial organization's decision to address minor 
worker injuries should be justified based on their own harm, not on the belief that the same interventions will control major hazards.

In regard to causal analysis, Heinrich's early, radical view in the 1940s was that the genesis of accidents is identical up and down the triangle. He wrote, "The predominant causes of no-injury accidents are, in average cases, identical with the predominant causes of major injuries, and incidentally of minor injuries as well" (Heinrich, 1941). Heinrich later equivocated, writing in 1980 that "perhaps circumstances that produce the severe accident are different from those that produce the minor accident." Manuele and others have further emphasized causal differences; e.g., "Causal factors for low-probability, high-consequence events are rarely represented in the analytical data on frequent incidents" (Manuele, 2014).

Hale (2002) echoes these criticisms of Heinrich both in regard to injury reduction and to causal understanding. He notes, however, that different industrial jobs and operations may be different in regard to the applicability of Heinrich's principles. Heinrich may be correct in regard to some types of hazards, but wrong in regard to others. Hale's prescribed course for industrial safety is to "assume that the causes are different until proven otherwise."

\section{HEINRICH'S CONCEPTS \& NATURALISTIC DRIVING}

Heinrich never studied traffic crashes but his ideas inspired a dominant early $21^{\text {st }}$ century traffic safety research methodology: Naturalistic Driving (ND). More specifically, Heinrich's theory of identical causal mechanisms is the foundational assumption underlying the ND Mixed-Safety Critical Event (SCE) method. Mixed-SCE datasets consist mostly of driver evasive maneuvers or other aberrant dynamic events captured using videos and other instrumentation. Unlike major crash datasets and indeed most empirical scientific datasets of all kinds, Mixed-SCE datasets are not derived from a target population sampling frame. Instead, ND researchers decide on the types of SCEs (e.g., swerves, lane breaks) to be included, and they control event type proportions by adjusting dynamic thresholds for each type. Thus, the Heinrich assumption of identical causal mechanisms (and therefore unlimited generalizability) is essential to the external validity of this method in relation to any defined crash outcome. This was expressed in a validation study (Guo et al., 2010) of the 100-Car Naturalistic Driving Study:

- "The underlying assumption of Heinrich's Triangle is that the unsafe acts, minor injuries, and major injuries all share the same underlying causal mechanism."

- "One key requirement for using near-crashes as a surrogate [dependent] measure is that they possess the same causal mechanism as crashes (the only difference between a crash and an appropriate near crash surrogate is the severity of the safety outputs)."

This assumption is espoused in Mixed-SCE reports, such as those relating to truck driver Hoursof-Service rules; e.g., "The non-crash events [SCEs] were operationally defined for this study as having elements identical to a crash scenario, with the exception that a successful evasive maneuver was also present" (Blanco et al., 2016, P. 184). Within these statements is the implicit assumption that crashes avoided (i.e., SCEs) are representative of crashes which actually occur.

Empirical science ordinarily identifies an accessible external target population (e.g., breast cancer, gun deaths, asleep-at-the-wheel crashes) and draws cases directly from that population 
using structured, algorithmic sampling (Privatera, 2014). This ensures external validity within the accuracy of sampling procedures. The ND M-SCE method does not identify or sample from a target population of actual crashes, but rather assumes that crashes (and high-acceleration events) throughout the population are causally identical and thus can be represented by ad hoc combinations of errant driving events.

A 100-Car validation study (Guo et al., 2010) presented evidence of crash-near crash similarities by comparing the data element percentages for different coding variables. Yet Knipling (2017) has found significant dissimilarities within the study's data. For example, the Conflict Type variable described how crashes occurred using categories like single-vehicle, conflict with lead vehicle, conflict with adjacent vehicle, pedestrian, etc. Crash and near-crash Conflict Type profiles correlated only $+0.44\left(\mathrm{R}^{2}=.20\right)$. The correlation between crashes and incidents (the lowest SCE category) was also $+0.44\left(\mathrm{R}^{2}=.20\right)$. In contrast, the near-crash correlation with incidents was $+0.96\left(\mathrm{R}^{2}=.93\right)$. Thus, crashes were distinctly different than non-crashes in regard to how they occurred. Single-vehicle scenarios (including object/obstacle and parked vehicle) were $37 / 69$ crashes (54\%), versus just 59/761 near-crashes (8\%). Odds ratio: 13.8. This single- versus multi-vehicle disparity alone is here considered sufficient evidence to disprove near-crash vs. crash representativeness. Single- and multi-vehicle crash involvements are wellknown to have sharply discrepant causal profiles, as explained below.

\section{HORIZONTAL HETEROGENEITY}

Crash involvements occur in many different ways. Causal profiles of crashes are associated with profiles of where, when, and how they occur. "Horizontal" heterogeneity refers to the variety of types and roles seen within any population of crashes or involvements. This is analogous to the heterogeneity of political and social opinions seen as a function of gender, age, race, and other personal variables. In both cases, samples must be representative of their target populations if they are to yield valid inferences.

A particular causal schism is seen between the two main types of crash involvements, singlevehicle (SV) and multi-vehicle (MV). The National Motor Vehicle Crash Causation Study (NMVCCS) and Large Truck Crash Causation Study (LTCCS) were two in-depth crash investigation studies based on nationally representative sampling. Knipling (2017) disaggregated at-fault involvements from these two studies into SV and MV categories and into nine Critical Reason (CR) categories. The nine CR categories were: (1) non-performance (e.g., asleep); (2) inadequate surveillance; (3) other recognition failure; (4) too fast; (5) other decision failure; (6) maneuver execution error; (7) vehicle failure; (8) environmental/roadway; and (9) miscellaneous other. Below are the four Pearson $\mathbf{r}$ correlations comparing the $\mathrm{CR}$ profiles of each crash category within each study:

- NMVCCS SV $\times$ NMVCCS MV: $\mathbf{- 0 . 3 5}$

- LTCCS SV $\times$ LTCCS MV: $\mathbf{- 0 . 1 5}$

- $\quad$ NMVCCS SV $\times$ LTCCS SV: $+\mathbf{0 . 6 7}$

- NMVCCS MV $\times$ LTCCS MV: $+\mathbf{+ 0 . 7 5}$.

The two negative correlations show that, in regard to causal profiles, SV and MV involvements were more dissimilar than similar. This finding reinforces the need for representative sampling 
from a target crash population; any sampling bias favoring SVs or MVs would distort prevalence estimates of causes. The two positive correlations suggest causal robustness within each crash category. These high within-type, between-study correlations were seen even though NMVCCS and LTCCS involved different vehicle types, driver demographics, and data collection years.

Eighty-one percent (81\%) of Blanco's 2016 truck SCEs were SV. In contrast, only 21\% of 2016 police-reported large truck crash involvements were SV (FMCSA, 2018). The disparity between these percentages combined with SV-MV causal dissimilarities (as shown above) suggest extreme SCE causal unrepresentativeness in relation to truck crashes.

Figure 1 depicts the distributions of five causal factors (four CRs + alcohol, a contributing factor) for four at-fault crash involvement types from NMVCCS. Each crash involvement type shows a distinctive causal pattern. Even the two rear-end subtypes are notably different. Conversely, the prevalence of the five factors varies markedly across the involvement types. Note that the four CRs are mutually exclusive with each other but that alcohol as a contributing factor is not.

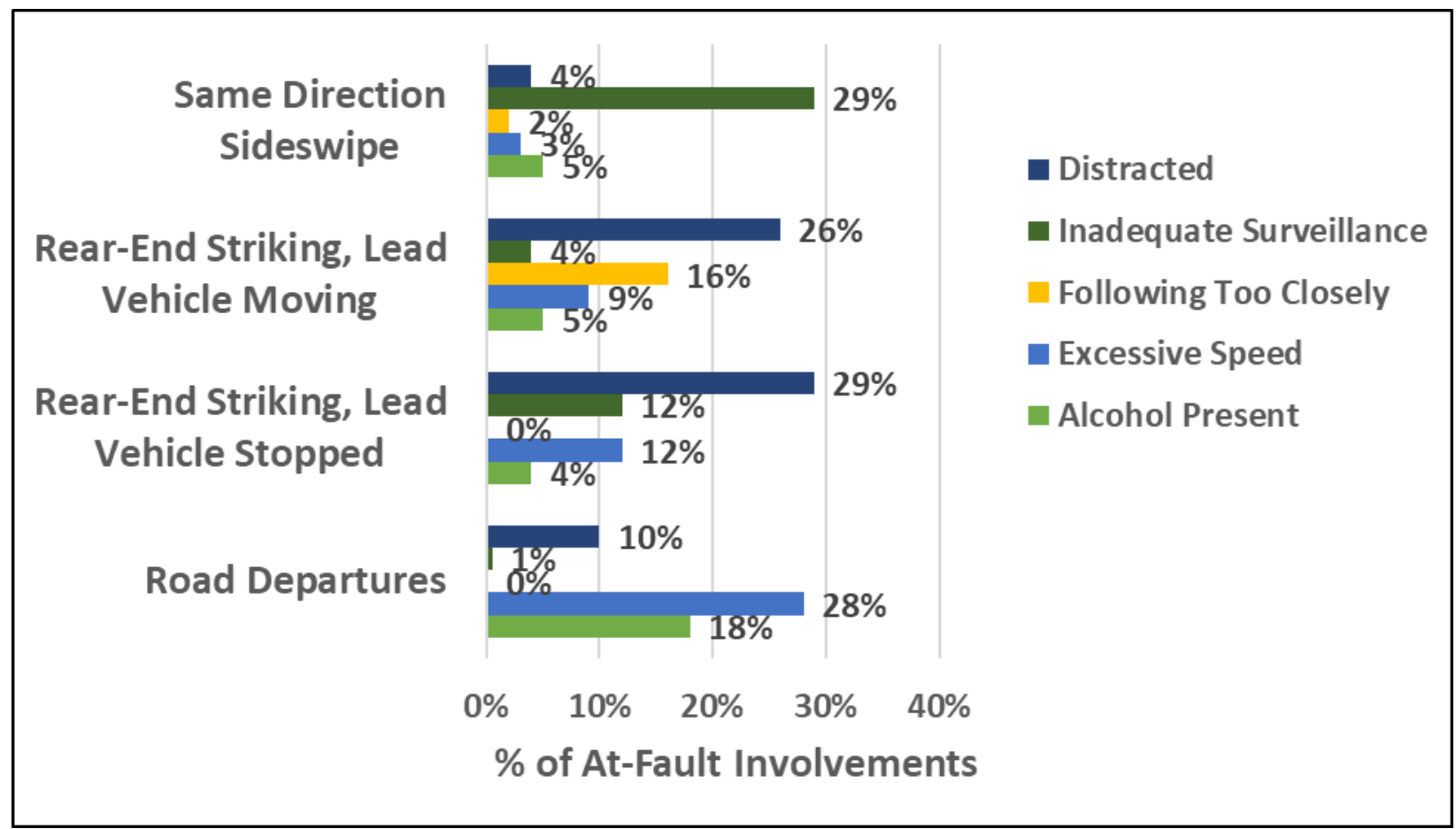

FIGURE 1. Weighted distributions of selected factors for four crash involvement types in NMVCCS (Knipling, 2017). Distraction includes internal and external. Excessive speed includes excessive speed for conditions, to be able to respond, and for a curve/turn. Alcohol percentages exclude unknowns/unreporteds.

\section{VERTICAL HETEROGENEITY}

Vertical heterogeneity refers to changes in crash profiles across different severity levels. For example, the AAA Safety Foundation (Tefft, 6 ) estimated that passenger vehicle drivers were drowsy in 3\% of drivers of no-injury crashes, $8 \%$ of injury crashes, and $15 \%$ of fatal crashes. An Australian study (Wundersitz \& Balcock, 2011) found that $66 \%$ of fatal car crashes involved extreme or illegal behaviors, versus just $18 \%$ of non-fatal crashes. In Europe, af Wåhlberg has 
found, in data to be published, that the causes and other characteristics of Swedish city bus crashes vary significantly by crash severity. Of 17 characteristics studied, 11 had statistically significant differences by severity.

NHTSA (Blincoe et al., 2015) reported the presence of six factors in crashes of eight different severity levels ranging from Property Damage Only (PDO) to fatal. Figure 2A shows percentages of three crash characteristics (single vehicle, rural, and two-lane roadway) as a function of severity. One sees sharp rises in each of these. Figure 2B shows the same NHTSA estimates for three independent causation-related factors: alcohol, excessive speed, and distraction. Alcohol and speed-related rise markedly, while driver distraction followed a different pattern. All of these examples teach us that crashes cannot be reliably characterized without reference to specific severity levels. Quantitative statements about crash characteristics and causes require a specified target crash population referent in order to be meaningful.

Vertical heterogeneity is also seen in crashes observed directly in ND studies. SHRP2 was the first ND study to capture enough crashes for reliable analysis. The Insurance Institute for Highway Safety (Kidd \& McCartt, 2015) provided severity distributions of SHRP2 crash involvement characteristics. Rear-end and road departure were the predominant crash types, and their proportions varied sharply by severity. Rear-end scenarios were $54 \%$ of the 98 most severe crashes, but only $12 \%$ of the 597 minor contacts. They are about one-third of police-reported crashes nationally. Road departures were only $12 \%$ of severe crashes but $64 \%$ of minor ones. IIHS concluded that SHRP2 crash severity and crash type distributions differed substantially from crashes reported to police which form the basis of national assessments of the motor vehicle crash problem. Only 7\% of SHRP2 crashes had significant injury potential (based on Delta-Vs), and another 10\% were "police-reportable." These low percentages further belie the analytic risk inherent in ND extrapolations to the national crash picture per societal thresholds. Sensor systems detecting all impacts capture many events not considered consequential by individual drivers and society (Knipling, 2017). Moreover, the human consequences of crashes reside predominantly at the very highest levels of severity. NHTSA (Blincoe et al., 2015) estimates that the $22 \%$ of U.S. crashes resulting in injuries and fatalities represent $91 \%$ of all crash harm measured as economic and human quality-of-life effects. Representativeness in relation to serious crashes seems essential for any dataset claiming high safety relevance.

\section{CONCLUSION: THE HETEROGENEITY PRINCIPLE}

Motor vehicle crashes are pervasively heterogeneous in regard to both objective characteristics (when, where, and how they happen) and in regard to causation (why they happen). Thus, extrapolations from one category of crashes (or from non-crash SCEs) to other target crash populations are likely to be spurious. This Heterogeneity Principle should replace Heinrich's theory of "identical mechanisms" as the starting assumption and guiding tenet of any attempt to extrapolate crash or ND findings to other target crash populations. Two dimensions of heterogeneity can be conceptualized. Crash categories are horizontally heterogeneous in regard to how and why they happen. Crashes are also vertically heterogeneous. The prevalence of their causal factors and other characteristics varies markedly by severity. The historic "Heinrich Law" positing identical causal mechanisms across accidents of different severities is simply not true in regard to traffic crashes. Heinrich's Law has been an implicit or explicit rationale for studies of 
crash risk or causation which do not specify target crash populations but rather assume wide generalizability of their findings. A conclusion here is that any quantitative statement about crash risk from any factor is incomplete and possibly spurious without an accompanying crash population referent and evidence of representativeness in relation to that specific referent. Lack of external validity in relation to important crash populations has been a particular deficiency of ND Mixed-SCE datasets. And this is also likely true for ND all-crash datasets which record all physical contacts but do not impose event severity thresholds reflective of real harm.
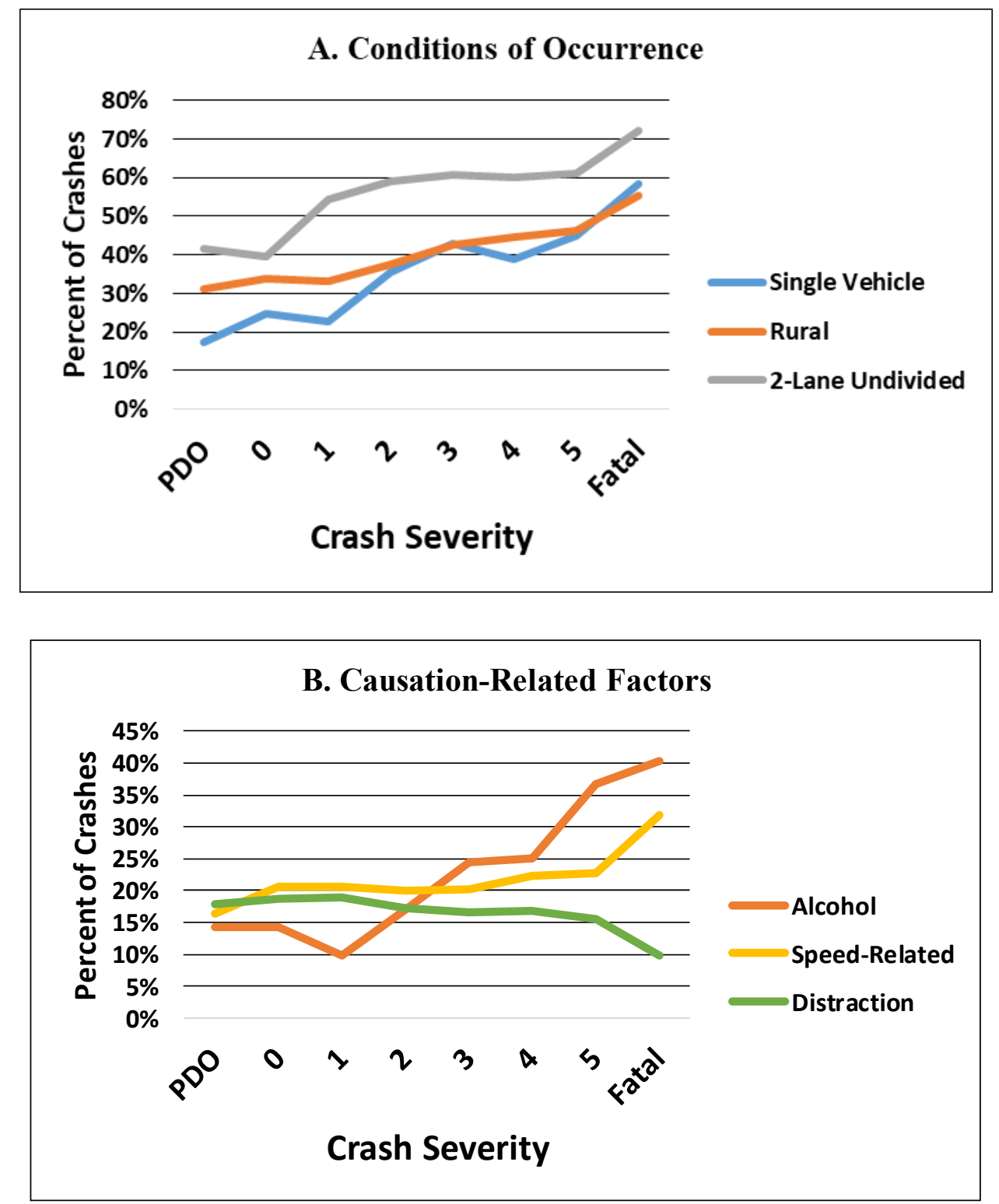

Figure 2. Trends in crash (a) conditions of occurrence and (b) causation-related factors with increasing severity (drawn from data in Blincoe et al., 2015). Multiple descriptors can apply to each crash. 


\section{REFERENCES}

Af Wåhlberg, A.E., (2008) Driver celeration behaviour and accidents - an analysis. Theoretical Issues In Ergonomics Science, 9:5, 383-403, Available At:

Http://Dx.Doi.Org/10.1080/14639220701596722.

Blanco, M. J. S. Hickman, R. L. Olson, J. Bocanegra, R. J. Hanowski, A. Nakata, M. Greening, P. Madison, G. T. Holbrook, and D. Bowman. (2016). Investigating critical incidents, driver restart period, sleep quantity, and crash countermeasures in commercial vehicle operations using naturalistic driving data collection. FMCSA-RRR-13-017. Wash. DC: DoT.

Blincoe, L. J., Miller, T. R., Zaloshnja, E., \& Lawrence, B. A. (2015) The economic and societal impact of motor vehicle crashes, 2010 (revised). DOT HS 812 013, NHTSA.

Dunlop, L. (2013). Beyond the safety triangle. Safety \& Health Practitioner, 31, (10), 45-46. Available at: https://www.shponline.co.uk/beyond-the-safety-triangle/.

Guo, F., S. G. Klauer, M. T. McGill, and T. A. Dingus. Evaluating the relationship between near-crashes and crashes: can near-crashes serve as a surrogate safety metric for crashes? DOT HS 811 382, NHTSA, October 2010.

Hale, A. (2002). Conditions of occurrence of major and minor accidents: urban myths, deviations and accident scenarios. Jaffelaan 5, 2628 BX Delft: Tijdschrift voor toegepaste Arbowetenschap. Https://j3corp.net/wp-content/uploads/2018/03/2002-03-hale-full-paper-trf.pdf

Heinrich, H.W. (1941) Industrial accidents prevention: a safety management approach, $2^{\text {nd }}$ Edition. McGraw Hill, New York: McGraw-Hill. Also $5^{\text {th }}$ Edition (1980).

Kidd, D. G. \& McCartt, A.T. (2015) The relevance of crash type and severity when estimating crash risk using the SHRP2 naturalistic driving data. 4th Int. Driver Distraction and Inattention Conference, Sydney, New South Wales.

Knipling, R.R. (2017) Crash heterogeneity: implications for naturalistic driving and for understanding crash risks. Paper 17-02225, Session 247, TRB Annual Meeting, Washington DC. Published in Transportation Research Record No. 2663.

Manuele, F. A. (2014) Heinrich revisited: truisms or myths, 2nd edition. National Safety Council, Itasca, IL, 2014.

Manuele, F.A. (2008) Serious injuries \& fatalities; a call for a new focus on their prevention. Professional Safety, Pp. 32-39, December 2008.

Pradhan, A.K., B.T. Lin, C. Wege, and F. Babel. (2017) Effects of behavior-based driver feedback systems on commercial long haul operator safety. Proceedings of the 9th International Driving Symposium on Human Factors in Driver Assessment, Training, and Vehicle Design, Manchester Village VT, June 26-29, 2017.

Privitera, G. J. (2014) Research methods for the behavioral sciences, Sage Publications, Inc.

Tefft B.C. (2012) Prevalence of motor vehicle crashes involving drowsy drivers, United States, 1999-2008. Accident Analysis \& Prevention, Vol. 45, No. 1, 180-186.

Wundersitz, L. and M. Baldock. (2011) The relative contribution of system failures and extreme behaviour in South Australian crashes, Publication CASR092, Centre for Automotive Safety Research, University of Adelaide. 